\title{
WAVELET- FuZZY BASED MULTI TERMINAL Transmission System PROTECTION SCHEME IN The Presence Of Statcom Controller
}

\author{
J.Uday Bhaskar ${ }^{1}$ and S.S Tulasi Ram ${ }^{2}$ \\ ${ }^{1}$ Department of Electrical \& Electronics Engineering, D.M.S S.V.H College Of \\ Engineeing, Machilipatnam, India \\ ${ }^{2}$ Department of Electrical \& Electronics Engineering, University College Of Engineeing- \\ Jntuh, Hyderabad, India
}

\begin{abstract}
In This Paper, A New Protection Scheme In The Areas Of Accurate Fault Detection, Classification And Location Estimation For Multi Terminal Transmission System Compensated With Statcom Is Proposed. The Fault Indices Of All The Phases At All The Terminals Are Obtained By Analyzing The Detail Coefficients Of Current Signals Through Bior 1.5 Mother Wavelet. The Complete Digital Simulation Of A Transmission System With Statcom Is Performed Using Matlab /Simulink For Fault Detection, Classification, And Faulty Terminal Identification With Variations In Fault Distance And Fault Inception Angle For All Types Of Faults And Fuzzy Inference System Is Used To Estimate The Fault Location. The Protection Scheme Yielded Accurate Results Within Half Cycle And Show That The Above Scheme Is Suitable For Multi Terminal Transmission System With And Without Statcom Compensation.
\end{abstract}

\section{KEYWORDS}

Multi Terminal Transmission System, Wavelet Transform, Threshold Value, Statcom, Fault Inception Angle, Fault Indices, Fuzzy Logic

\section{INTRODUCTION}

The Difficulties In Obtaining The Right-Of-Way And The Increase In The Capital Expenditure Have Led To The Development Of Multi Terminal Transmission Lines Where More Than Two Terminals Are Interconnected. The Protection Of Such Systems Is Difficult As Compared With Two-Terminal Systems. The Multi Terminal Lines Experience Problems Generated By The Intermediate In Feed Of The Currents From The Other Terminals Or An Out Feed To The Terminals, Variations In Section Lengths And Source Impedances And Superimposing Of Currents Which Require The System To Be Protected Under Fault Conditions. The Multi Terminal Transmission Lines Are Usually Compensated With Facts Devices Like Svc, Tcsc, Upfc, Statcom Etc., For Transmission Efficiency And To Make Necessary Corrections Of Transmission Functionality. Power Flow Along The Transmission Lines Needs To Be Controlled And Facts Devices Received Some Attention As They Can Alter Power System Parameters In Order To Control Power Flow. The Accurate Fault Detection And Classification Are Vitally Important For Efficient Transmission As The Faults Cause Interruption Of Power Flow. Quick Detection Of Faults Helps In Faster Maintenance And Restoration Of Supply Which Results In Improved Economy And Power Supply Reliability. The Statcom Is An Important Member Of Facts Family And Is Capable Of Enhancing The Power Transfer Capability With Apparent Impedance Being Influenced By The Absorption Or Injection Of The Reactive Power Causing 
Under Reaching Or Over Reaching Of Distance Relay Wavelet Transform Is An Effective Tool In Analyzing Transient Current Signals Associated With Faults Both In Frequency And Time Domain. Amany Et Al Have Done The Work Related With Dwt And Wavelet Entropy To Analyse Fault Current Signals When Facts Device Placed In The Mid Point Of The Two Terminal Transmission Line [1].Gorakhnath Abande Et Al Have Given The Impact Analysis Of Statcom On Distance Relay When Statcom Is Placed At Different Locations During Phase To Ground Fault[2]. R.Illango Et Al Proposed That Statcom Has Great Impact On The Measured Impedance At The Relaying Point Which In Turn Effects The Performance Of The Relay. With Usage Of Synchronized Data Sampling Of Statcom Current At The Relay Location[3].Sriteja Et Al Have Emphasized The Effect Of Statcom On Distance Relay Performance In A Transmission Line[4]. A.Y.Abdelaziz Et Al Have Done Work By Extracting The Modal Information From The Measured Signals To Classify The Faults In A Facts Compensated Transmission Line[5].Qazi Waqar Ali Et Al Have Applied Facts Devices To Power System And Found That Satisfactory Regulation Of System Voltage And Increased Loading Ability[6].Sham M.V Et Al Proposed The Distance Relay Performance In The Presence Of Statcom[7].E.Kazeni Abharian Et Al Emphasized The Effective Power Flow Control With Statcom Controller Than With Pi, Pid Controllers[8].R.P.Hasabe Et Al Applied Neural Networks To Locate Faults Along The Transmission Line[9.]Majid Jamil Et Al Proposed The Fault Location Estimation Using Wavelet And Ffnn Methods For Two Terminal Transmission System[10].

E.Latha Mercy Et Al Applied Dwt And Anfis Techniques For Fault Detection And Classification In Transmission Lines With High Fault Resistance[11] Bo,Z.Q Proposed A Protection Scheme For Multi-Terminal Transmission Circuits Such As Unit And Non-Unit Schemes. The Unit Schemes Require Extensive Communication Channels Between The Line Ends[12]. Bhalija,B Et Al Proposed A Distance Relaying Scheme To Detect High Resistance Faults On Two Terminal Transmission Lines[13]. Brahma And Girgis Proposed A Fault Location Scheme For A MultiTerminal Transmission Line Using Synchronized Voltage Measurements At All Terminals[14]. Lyonette,D.R.M Et Al Proposed A Different Directional Comparison Techniques For MultiTerminal Lines, Which Compare The Polarity Of Fault Generated Transient Current Signals[15]. B.Bhalija Et Al Proposed A Differential Protection Scheme For Tapped Transmission Lines Where Outfeed Current In Case Of Internal And External Faults Was Considered[16] .Al-Fakhri Proposed Differential Protection Scheme For Multi-Terminal Lines Using Incremental Currents [17]. Yugant A Parate Et Al Investigated The Enhancement Of Power System Stability Using Facts Devices[18].Joe -Air Jiang Et Al Proposed A Protection Scheme For Fault Detection, Direction Discrimination, Classification And Location In Transmission Lines[19]. Ching-Shan Chen Et Al Considered Double And Three Terminal Lines For Accurate Fault Detection Using Phasor Measurement Units[20]. Semwal Et Al Used Fuzzy Inference System With Two Crispy Values As Inputs And Reduced Number Of Fuzzy Rules Where The Approach Is Fast And Effective [21]. There Must Be Some Innovative Methods To Be Developed For Multi Terminal Transmission Line Protection. In This Paper, Wavelet MultiResolution Analysis Is Used For Detection And Classification Of Faults And Faulty Terminal Identification On Four Terminal Transmission System. Fuzzy Inference System Has Been Applied For Fault Location Estimation. Detail D1 Coefficients Of Current Signals At All The Four Ends Are Used To Detect And Classify The Faults. The Current Signals Are Analyzed Taking Into Consideration That Sum Of The Current Coefficients At All The Four Terminals. The Major Contribution Of This Paper Is The Fast Detection Of Faults And Faulty Terminal Identification Which Is Very Important In Present Day's Power System Protection. The Faulty Terminal Identification Is Done By Considering The Fault Indices Of The Faulty Phase At All The Terminals And The Fault Index Of The Faulty Terminal Is Maximum As Compared With The Fault Indices Of The Same Phase At The Remaining Terminals Which Is Very Novel Feature In Terminal Identification. 


\section{WAVELET ANALYSIS}

Wavelet Transform (Wt) Is An Efficient Means Of Analyzing Transient Currents And Voltages. Unlike Discrete Fourier Transform, Wt Not Only Analyses The Signal In Frequency Bands But Also Provides Non-Uniform Division Of Frequency Domain I.E. Wt Uses Short Window At High Frequencies And Long Window At Low Frequencies. This Helps To Analyze The Signal In Both Frequency And Time Domains Effectively. A Set Of Basis Functions Called Wavelets, Are Used To Decompose The Signal In Various Frequency Bands, Which Are Obtained From A Mother Wavelet By Dilation And Translation Hence The Amplitude And Incidence Of Each Frequency Can Be Found Precisely. Wavelet Transform Is Defined As A Sequence Of A Function $\{\mathrm{H}(\mathrm{N})\}($ Low Pass Filter) And $\{\mathrm{G}(\mathrm{N})\}$ (High Pass Filter). The Scaling Function $\omega(\mathrm{T})$ And Wavelet $\Psi(\mathrm{T})$ Are Defined By The Following Equations.

$$
\begin{aligned}
& \varphi(t)=\sqrt{2 \sum h(n) \varphi(2 t-n)}, \\
& \psi(t)=\sqrt{2 \sum g(n) \varphi(2 t-n)}
\end{aligned}
$$

Where $\mathrm{G}(\mathrm{N})=(-1) \mathrm{N} H(1-\mathrm{N})$. A Sequence Of $\{\mathrm{H}(\mathrm{N})\}$ Defines A Wavelet Transform. This Method, Like The Fourier Transform, Provides Information Related To The Frequency Composition Of A Waveform, Thus It Is More Appropriate Than The Familiar Fourier Methods For The NonPeriodic, Wide-Band Signals Associated With Electromagnetic Transients. Wavelet Transform Provides A New Tool For Signal Processing In Contrast To The Traditional Fourier Analysis That Averages Frequency Features Both In Time And Frequency. Wavelets Allow The Decomposition Of A Signal Into Different Levels Of Resolution Which Gives A Much Better Signal Characterization And A More Reliable Discrimination. The Multi Resolution Analysis Of Wavelet Can Be Utilized Effectively In Analyzing The Power System Transients. The Feature Extraction Property Of Wavelets Transforms Is Exploited In The Area Of Protection Of Transmission Line To Detect And Classify The Faults On Various Components. There Are Many Types Of Wavelets Such As Haar, Daubachies, Symlet, Bior Etc. The Selection Of Mother Wavelet Is Based On The Type Of Application And After Extensive Work With All Wavelets, Bior-1.5 Was Found To Be Effective And Has Been Selected As Mother Wavelet.

\section{Fault Detection And Classification}

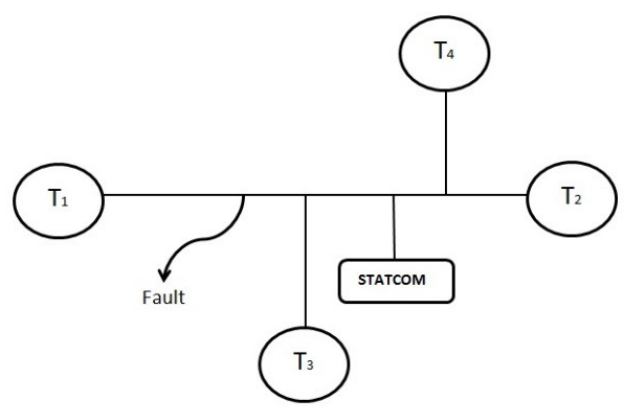

Fig.1.Single Line Diagram of the Proposed Four Terminal Transmission System. 
Figure 1 Shows The Single Line Diagram Of The Multi Terminal Transmission System Considered For The Proposed Scheme. Four 110-Km $400 \mathrm{Kv}$ Transmission Lines Compensated With 100mva Static Synchronous Compensator (Statcom) At The Middle Of The Second Terminal Are Interconnected. The Scheme Is Evaluated Using 400kv, 50hz Four Terminal Transmission System Whose Line Parameters Are $\mathrm{R}_{0=} 0.1888 \omega / \mathrm{Km}$, $\mathrm{R}_{1}=0.02 \omega / \mathrm{Km}, \mathrm{L}_{0}=3.5 \mathrm{mh} / \mathrm{Km}, \mathrm{L}_{1}=0.94 \mathrm{mh} / \mathrm{Km}, \mathrm{C}_{0}=0.0083 \mu \mathrm{f} / \mathrm{Km} ., \mathrm{C}_{1}=0.012 \mu \mathrm{f} / \mathrm{Km} . \mathrm{A}$ Sampling Frequency Of 16khz Is Chosen To Capture The High Frequency Content Of Current Signals.The System Is Modeled In Matlab Simulink Environment. The Network Is Simulated For Various Fault Situations. Exhaustive Simulations Were Carried Out For L-G, L-L-G, L-L-L Faults Occurring At Different Locations Along The Paths Of Terminal 1 To Terminal 2, Terminal 2 To Terminal 3, Terminal 3 To Terminal 1, And Terminal 4 To Terminal 1. For Each Type Of Fault At A Particular Location, The Fault Inception Angle Was Varied To Evaluate The Performance Of The Proposed Scheme. Influence Of Fault Resistance Also Being Considered With Value Of 5 Ohms. Synchronized Sampling Of Three Phase Currents At All Terminals Was Carried Out And The Detail D1 Coefficients Were Used For Detection And Classification Of The Type Of Fault. The Three Phase Currents Of The Local Terminal Are Analyzed With Bior1.5 Mother Wavelet To Obtain The Detailed Coefficients $\left(\mathrm{D} 1_{1}\right)$ At Terminal 1 Over A Moving Window Of Half Cycle Length. These $\mathrm{D} 1_{1}$ Coefficients Are Then Transmitted To The Remote End. The Detailed Coefficients Received From The Remote End At Bus2 $\left(\mathrm{D}_{2}\right)$ Are Subtracted From The Local Detail Coefficients $\left(D 1_{1}\right)$ To Obtain Effective D1 Coefficients $\left(D 1_{e}\right)$. The Fault Index $\left(I_{f 1}\right)$ Of Each Phase Is Then Calculated As $I_{\mathrm{f} 1}=\Sigma\left|\mathrm{D} 1_{\mathrm{e}}\right|$
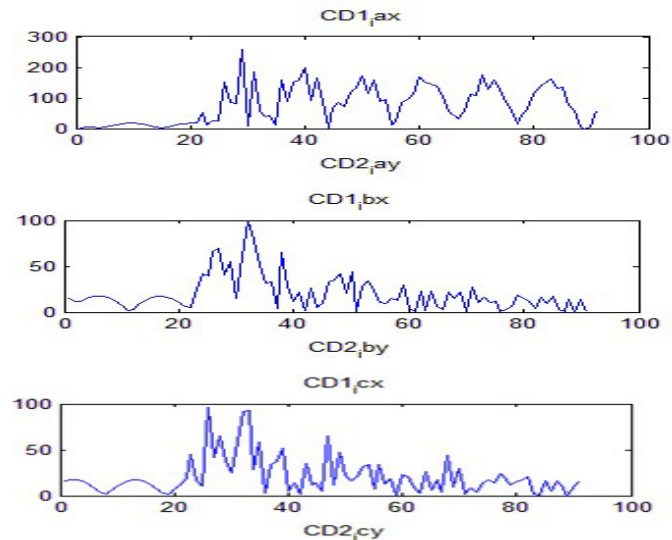

Fig. 2a.Three Phase Currents Ia,Ib,Ic at All Terminals for A-G Fault at Terminal-1
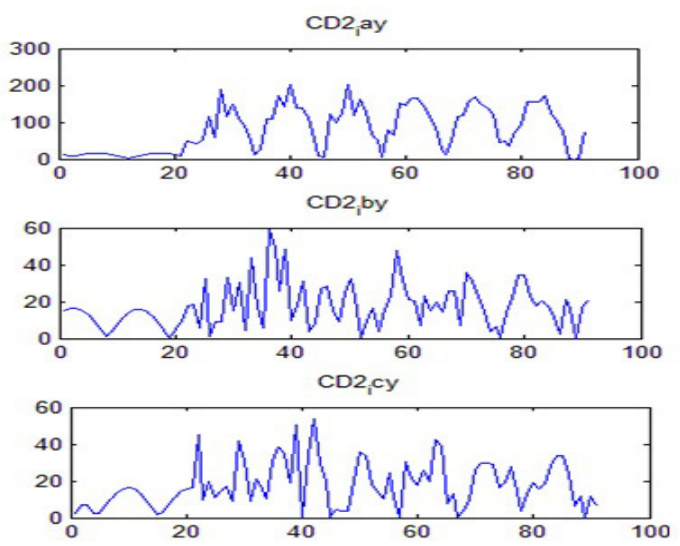

Fig. 2b.Three Phase Currents Ia,Ib,Ic at All Terminals for A-G Fault at Terminal-2 

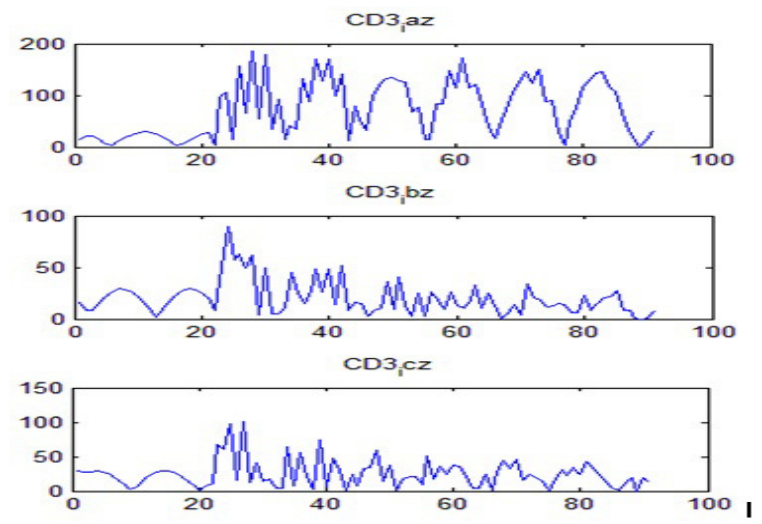

Fig. 2c.Three Phase Currents Ia,Ib,Ic at All Terminals for A-G Fault at Terminal-3
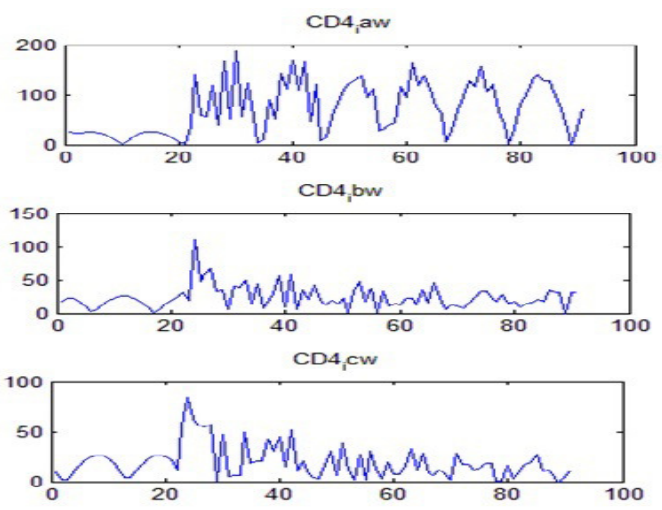

Fig. 2d.Three Phase Currents Ia,Ib,Ic at All Terminals for A-G Fault At Terminal-4

The Performance Of The Scheme In Detecting And Classifying The Faults I.E. Line-To-Ground, Line-To-Line, Line-Line-To- Ground, Triple-Line-To- Ground Is Evaluated. In All The Cases Studied, The Scheme Is Able To Detect The Faults. The Fault Inception Angle Is Varied From $20^{\circ}$ To $180^{\circ}$ For All Types Of Faults. The Simulations Show That The Fault Inception Angle Has A Considerable Effect On The Phase Current Samples And Therefore On Wavelet Transform Output Of Post-Fault Signals.

Digital Protection Scheme Involves The Analysis Of Current Signals Of All The Three Phases At All The Terminals. The Impedance Measurement Involves Both The Voltage And Current Signals To Be Analyzed And Takes More Time To Measure Faulty Section Impedance Thereby The Relay Operation. The Faulty Phases Will Be Identified With The Analysis Of Only Current Signals And Fault Clearing Time Will Be Less Than Half Cycle. The Digital Protection Scheme Involved The Comparison Of All Phases With Threshold Value, Where The Faulty Phase(S) Indices Have Higher Value Compared With The Threshold Value And The Healthy Phase Indices Are Less Than The Threshold Value And Therefore The Digital Values Of The Fault Indices Play A Significant Role In The Protection Of Multi Terminal Transmission System. 
International Journal of Fuzzy Logic Systems (IJFLS) Vol.6, No.3, July 2016

\begin{tabular}{|r|l|r|r|r|l|l|}
\hline $\begin{array}{l}\text { Distance, } \\
\text { Km }\end{array}$ & Ia & Ib & Ic & Ia & Ib & Ic \\
\hline 10 & 1033.331 & 286.459 & 149.4982 & 1024.458 & 339.8103 & 165.9121 \\
\hline 20 & 998.2832 & 285.2209 & 151.271 & 990.3503 & 344.1673 & 144.6448 \\
\hline 30 & 973.8581 & 312.6837 & 162.4574 & 953.7788 & 329.2317 & 155.1531 \\
\hline 40 & 953.4877 & 294.3952 & 168.2564 & 922.266 & 343.1482 & 147.2432 \\
\hline 50 & 949.1886 & 281.5716 & 153.9101 & 920.6146 & 366.1937 & 122.6623 \\
\hline 60 & 917.093 & 285.6333 & 199.9271 & 855.2354 & 358.5313 & 162.3399 \\
\hline 70 & 888.4642 & 302.1282 & 148.8825 & 857.9772 & 327.5753 & 160.4146 \\
\hline 80 & 859.9076 & 298.6013 & 146.1221 & 828.838 & 327.076 & 149.7437 \\
\hline 90 & 853.2456 & 284.426 & 153.5551 & 831.3929 & 364.1094 & 131.7178 \\
\hline 100 & 818.1098 & 279.9247 & 187.2792 & 767.9162 & 301.783 & 168.2288 \\
\hline
\end{tabular}

Table. 1. Variation of Fault Indices with Distance at A Fault Inception Angle of 100 and 40 Degrees for A-G Fault from Terminal-1

\begin{tabular}{|r|r|l|l|l|l|l|}
\hline $\begin{array}{l}\text { Distance, } \\
\text { Km }\end{array}$ & Ia & Ib & Ic & Ia & Ib & Ic \\
\hline 10 & 1057.573 & 961.7888 & 189.5009 & 807.4977 & 731.0871 & 210.9502 \\
\hline 20 & 1040.357 & 942.6548 & 189.4051 & 795.1843 & 718.3432 & 206.0904 \\
\hline 30 & 1022.145 & 924.1152 & 192.1158 & 780.1028 & 702.838 & 203.8661 \\
\hline 40 & 1004.8 & 908.4013 & 193.4396 & 766.5435 & 690.4211 & 208.1875 \\
\hline 50 & 987.0542 & 890.6561 & 186.3663 & 749.2409 & 672.203 & 205.3493 \\
\hline 60 & 982.7881 & 882.8934 & 192.9558 & 768.3603 & 687.8382 & 207.9141 \\
\hline 70 & 949.5369 & 848.9852 & 190.7618 & 706.7732 & 623.8897 & 207.8097 \\
\hline 80 & 947.7505 & 852.5902 & 190.9937 & 730.7641 & 658.3374 & 207.2141 \\
\hline 90 & 931.273 & 833.8187 & 187.6191 & 724.2885 & 649.0083 & 206.7211 \\
\hline 100 & 911.6705 & 814.6469 & 190.852 & 702.303 & 621.637 & 206.8226 \\
\hline
\end{tabular}

Table. 2. Variation of Fault Indices with Distance at A Fault Inception Angle Of 100 and 40degrees for A-B Fault from Terminal-1

\begin{tabular}{|r|l|l|l|l|l|l|}
\hline $\begin{array}{l}\text { Distance, } \\
\text { Km }\end{array}$ & Ia & Ib & Ic & Ia & Ib & Ic \\
\hline 10 & 1042.393 & 1094.961 & 183.2439 & 1023.031 & 853.5925 & 192.1528 \\
\hline 20 & 1020.911 & 1073.287 & 174.6212 & 991.3387 & 831.1754 & 213.6953 \\
\hline 30 & 1011.031 & 1052.098 & 184.06 & 971.3961 & 796.029 & 191.8168 \\
\hline 40 & 990.0857 & 1044.688 & 140.3366 & 943.7259 & 764.4818 & 161.3464 \\
\hline 50 & 956.8631 & 1020.847 & 222.2276 & 879.2248 & 768.6352 & 250.2213 \\
\hline 60 & 951.8511 & 1013.83 & 132.2794 & 907.0141 & 761.2414 & 166.1711 \\
\hline 70 & 938.4284 & 960.0935 & 187.0728 & 854.9817 & 715.6742 & 215.5146 \\
\hline 80 & 938.6608 & 961.6798 & 170.9948 & 879.7436 & 686.7476 & 229.2 \\
\hline 90 & 912.7606 & 952.331 & 168.2597 & 848.7681 & 715.9616 & 227.6593 \\
\hline 100 & 929.5522 & 919.3 & 190.617 & 840.1008 & 688.9574 & 231.0944 \\
\hline
\end{tabular}

Table. 3. Variation of Fault Indices with Distance at A Fault Inception Angle Of 100 and 40 Degrees for A-B-C Fault from Terminal-1 


\begin{tabular}{|r|l|l|l|l|l|l|}
\hline $\begin{array}{l}\text { Distance, } \\
\text { Km }\end{array}$ & Ia & Ib & Ic & Ia & Ib & Ic \\
\hline 10 & 1052.235 & 1089.278 & 846.3662 & 1040.996 & 861.8766 & 1258.423 \\
\hline 20 & 1034.719 & 1069.696 & 826.4491 & 1026.869 & 844.7463 & 1240.881 \\
\hline 30 & 1016.948 & 1052.47 & 817.214 & 1004.081 & 829.7035 & 1212.627 \\
\hline 40 & 1000.253 & 1033.229 & 808.98 & 985.9953 & 814.1834 & 1198.16 \\
\hline 50 & 988.0762 & 1017.45 & 793.5142 & 981.739 & 796.0876 & 1160.313 \\
\hline 60 & 977.2336 & 988.448 & 664.2236 & 998.7082 & 741.3593 & 1148.663 \\
\hline 70 & 942.6669 & 1006.265 & 892.3341 & 877.5124 & 827.4979 & 1097.725 \\
\hline 80 & 947.7684 & 952.5956 & 786.6413 & 930.9151 & 796.9225 & 1042.324 \\
\hline 90 & 918.8919 & 946.2872 & 741.5403 & 957.866 & 734.4872 & 1112.003 \\
\hline 100 & 963.9481 & 942.8445 & 800.5099 & 950.4385 & 748.3551 & 1101.025 \\
\hline
\end{tabular}

Table. 4. Variation of Fault Indices with Distance at A Fault Inception Angle of 100 and 40 Degrees for A-B-G Fault from Terminal-1

\begin{tabular}{|r|l|l|r|l|r|r|}
\hline \multicolumn{1}{|l|}{ Fia } & Ia & Ib & Ic & Ia & Ib & \multicolumn{1}{l|}{ Ic } \\
\hline 20 & 1219.143 & 365.2023 & 169.4123 & 1035.732 & 406.9321 & 171.5462 \\
\hline 40 & 953.7788 & 329.2317 & 155.1531 & 831.3929 & 364.1094 & 131.7178 \\
\hline 60 & 800.7785 & 334.6379 & 183.6582 & 692.6818 & 355.6985 & 177.251 \\
\hline 80 & 807.5176 & 314.6352 & 179.618 & 690.6542 & 326.9855 & 169.9186 \\
\hline 100 & 973.8581 & 312.6837 & 162.4574 & 853.2456 & 284.426 & 153.5551 \\
\hline 120 & 1139.447 & 297.8991 & 200.5526 & 959.0316 & 275.3462 & 189.089 \\
\hline 140 & 964.7391 & 305.8995 & 171.2995 & 849.1141 & 276.384 & 177.962 \\
\hline 160 & 797.9094 & 236.4523 & 187.8835 & 700.0159 & 255.7934 & 161.936 \\
\hline 180 & 785.6498 & 257.8884 & 205.616 & 683.0886 & 278.4598 & 190.0645 \\
\hline
\end{tabular}

Table. 5. Variation Of Fault Indices With Fault Inception Angle For A-G Fault At A Distance Of 30 and $90 \mathrm{Km}$ From Terminal-1

\begin{tabular}{|r|l|r|l|l|l|r|}
\hline \multicolumn{1}{|l|}{ Fia } & Ia & Ib & Ic & Ia & Ib & Ic \\
\hline 20 & 937.8798 & 885.7476 & 228.9844 & 840.0977 & 792.0436 & 233.931 \\
\hline 40 & 780.1028 & 702.838 & 203.8661 & 724.2885 & 649.0083 & 206.7211 \\
\hline 60 & 718.5739 & 734.2625 & 191.0719 & 646.7307 & 665.3005 & 188.3375 \\
\hline 80 & 862.6578 & 849.658 & 219.7925 & 794.6285 & 786.462 & 215.1059 \\
\hline 100 & 1022.145 & 1024.115 & 192.1158 & 931.273 & 933.8187 & 187.6191 \\
\hline 120 & 890.1368 & 876.3922 & 176.7765 & 811.7434 & 798.9336 & 172.2426 \\
\hline 140 & 714.4459 & 735.1727 & 211.2695 & 653.692 & 674.9876 & 206.3764 \\
\hline 160 & 655.2473 & 751.1015 & 164.0773 & 598.2009 & 692.8947 & 163.8941 \\
\hline 180 & 808.4303 & 850.9312 & 188.5316 & 735.2762 & 777.4878 & 187.9613 \\
\hline
\end{tabular}

Table. 6. Variation Of Fault Indices With Fault Inception Angle For A-B Fault At A Distance Of 30 and $90 \mathrm{Km}$ From Terminal-1 
International Journal of Fuzzy Logic Systems (IJFLS) Vol.6, No.3, July 2016

\begin{tabular}{|r|l|r|l|l|l|l|}
\hline \multicolumn{1}{|l|}{ Fia } & Ia & Ib & Ic & Ia & Ib & Ic \\
\hline 20 & 1246.222 & 845.0209 & 194.8786 & 1088.736 & 734.8319 & 217.2859 \\
\hline 40 & 971.3961 & 796.029 & 191.8168 & 848.7681 & 715.9616 & 227.6593 \\
\hline 60 & 813.2102 & 924.1105 & 172.3566 & 703.5271 & 799.9258 & 178.4092 \\
\hline 80 & 832.2152 & 1147.935 & 217.5054 & 758.6075 & 984.504 & 184.3581 \\
\hline 100 & 1011.031 & 1052.098 & 184.06 & 912.7606 & 952.331 & 168.2597 \\
\hline 120 & 1166.752 & 850.4077 & 184.0081 & 1024.134 & 760.2768 & 156.9005 \\
\hline 140 & 977.4541 & 796.9471 & 168.2822 & 855.4737 & 717.8241 & 186.1562 \\
\hline 160 & 818.0573 & 913.0741 & 170.3418 & 722.7143 & 813.0054 & 166.5681 \\
\hline 180 & 817.8787 & 1166.265 & 145.7882 & 744.9672 & 1012.415 & 174.1026 \\
\hline
\end{tabular}

Table. 7. Variation Of Fault Indices With Fault Inception Angle For A-B-C Fault At A Distance Of 30 and 90 Km From Terminal-1
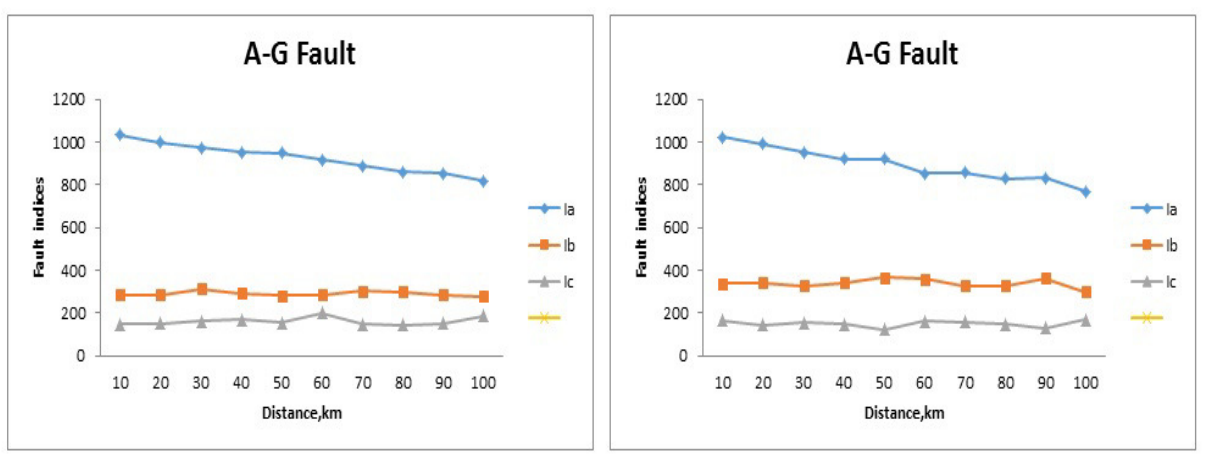

Figure 3. Variation Of Fault Indices With Distance At A Fault Inception Angle Of 100 And 40 Degrees For A-G Fault From Terminal-1
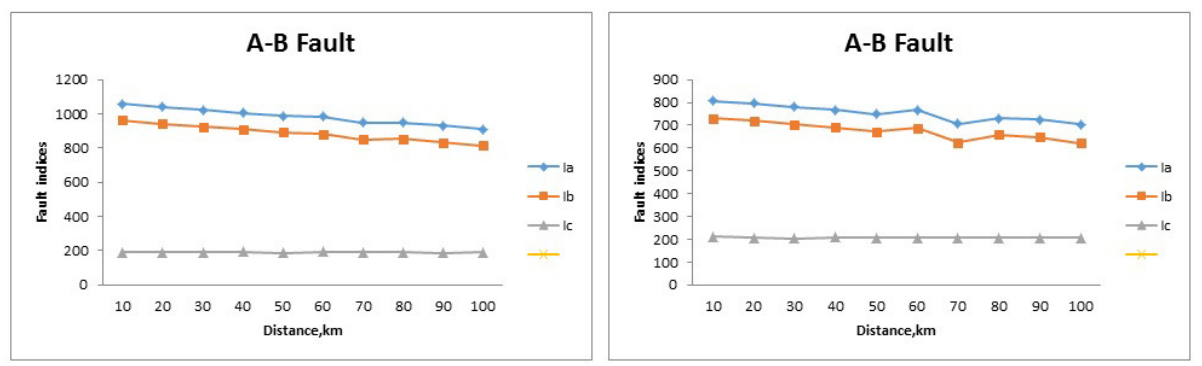

Figure 4. Variation Of Fault Indices With Distance At A Fault Inception Angle Of 100 And 40 Degrees For A-B Fault From Terminal-1
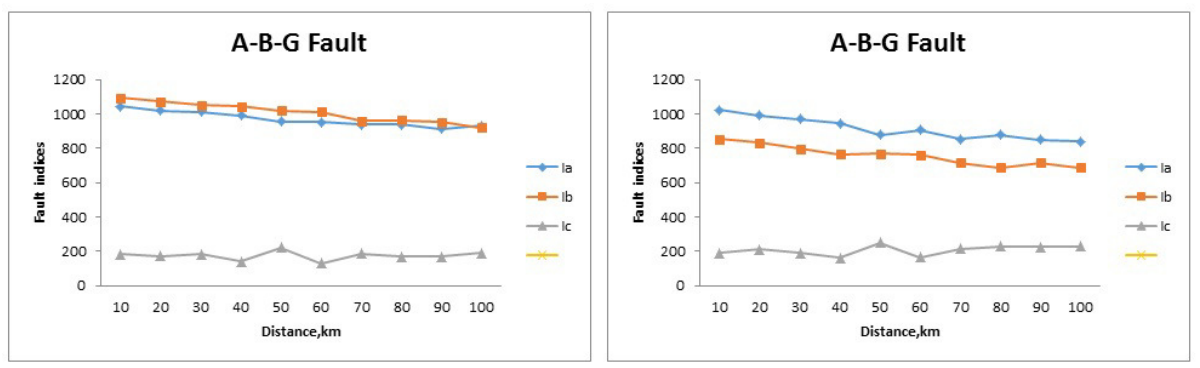

Figure 5. Variation Of Fault Indices With Distance At A Fault Inception Angle Of 100 And 40 Degrees For A-B-G Fault From Terminal-1 

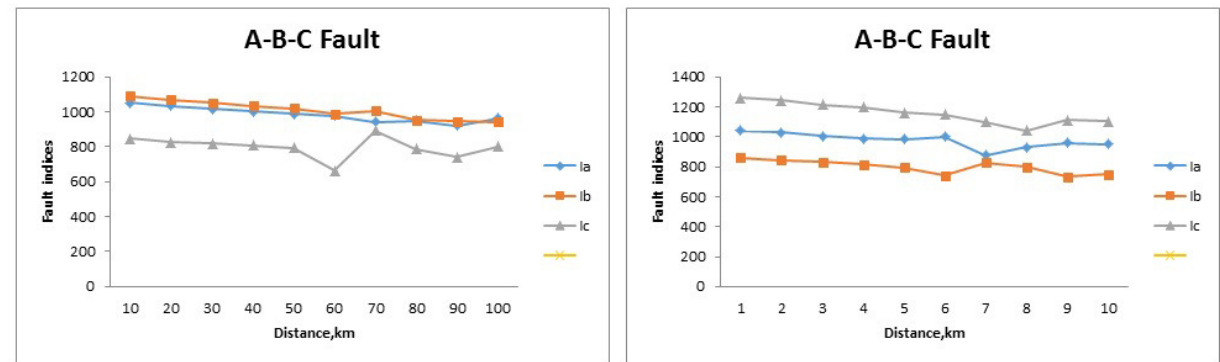

Figure 6. Variation Of Fault Indices With Distance At A Fault Inception Angle Of 100 And 40 Degrees For A-B-C Fault From Terminal-1
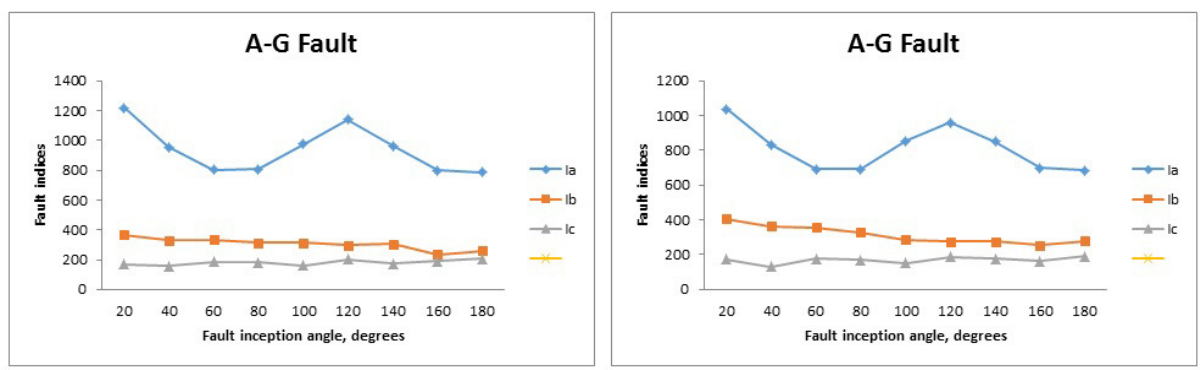

Fig. 7. Variation Of Fault Indices With Fault Inception Angle For A-G Fault At A Distance Of 30 And 90 Km From Terminal-1
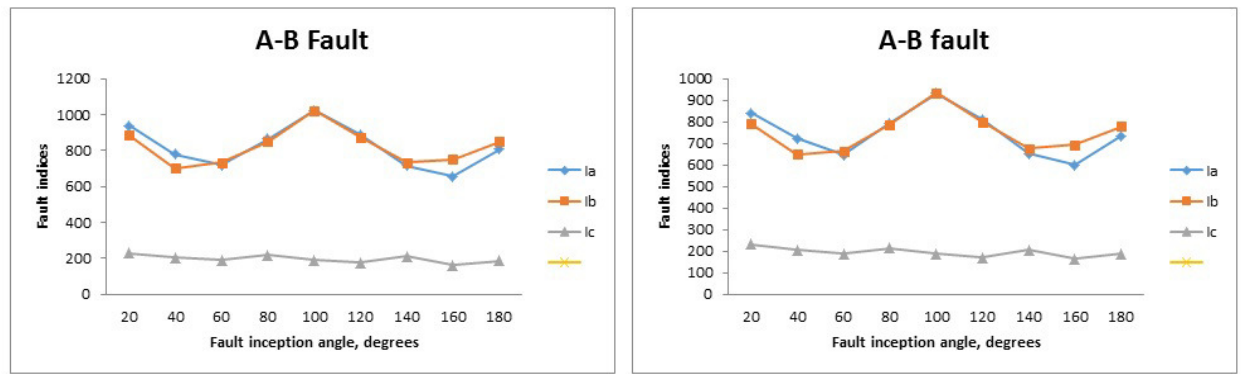

Fig. 8. Variation Of Fault Indices With Fault Inception Angle For A-B Fault At A Distance Of 30 And 90 Km From Terminal-1
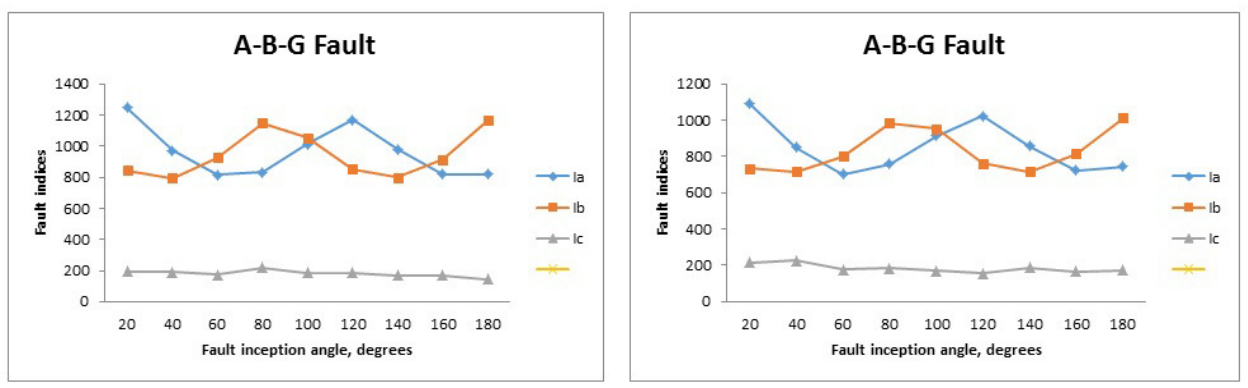

Fig. 9. Variation Of Fault Indices With Fault Inception Angle For A-B-G Fault At A Distance Of 30 And 90 Km From Terminal-1 

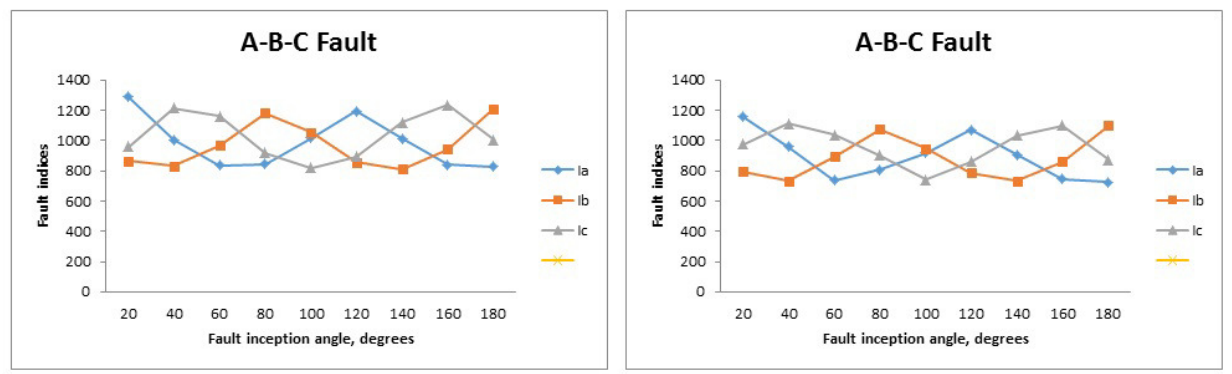

Fig. 10. Variation Of Fault Indices With Fault Inception Angle For A-B-C Fault At A Distance Of 30 \& 90 Km From Terminal-1

\section{FAULTY TERMINAL IDENTIFICATION}

The Faulty Terminals Are Identified By Comparing The Fault Indices At All The Terminals For The Same Fault And The Fault Index At The Faulty Terminal Has The Largest Value As Compared With Other Terminals Which Clearly Identifies The Faulty Terminal. The Identification For Different Faults Is Made By Considering The Variation Of Fault Indices With Fault Inception Angle And Distance.

\begin{tabular}{|r|l|l|l|l|l|l|l|l|}
\hline $\begin{array}{l}\text { Distance, } \\
\text { Km }\end{array}$ & T1 & T2 & T3 & T4 & T1 & T2 & \multicolumn{1}{l|}{ T3 } & T4 \\
\hline 10 & 1033.331 & 498.4475 & 574.7056 & 380.436 & 1024.458 & 484.9407 & 479.9955 & 452.6333 \\
\hline 20 & 998.2832 & 542.3093 & 588.2355 & 434.0846 & 990.3503 & 499.2732 & 487.246 & 472.8718 \\
\hline 30 & 973.8581 & 610.0538 & 632.7493 & 471.7781 & 953.7788 & 552.7896 & 507.7162 & 501.401 \\
\hline 40 & 953.4877 & 605.5186 & 636.3888 & 474.0194 & 922.266 & 601.381 & 532.3774 & 554.2876 \\
\hline 50 & 949.1886 & 592.9464 & 659.5619 & 436.3683 & 920.6146 & 663.6384 & 594.6475 & 576.1131 \\
\hline 60 & 917.093 & 635.1973 & 682.6034 & 458.3388 & 855.2354 & 628.3335 & 602.928 & 543.0156 \\
\hline 70 & 888.4642 & 676.7822 & 697.3325 & 529.642 & 857.9772 & 608.681 & 600.411 & 556.3666 \\
\hline 80 & 859.9076 & 711.1053 & 730.6986 & 536.8869 & 828.838 & 679.5285 & 639.4943 & 604.5029 \\
\hline 90 & 853.2456 & 732.317 & 761.3227 & 524.4016 & 831.3929 & 710.9502 & 675.0111 & 592.9964 \\
\hline 100 & 818.1098 & 757.0811 & 803.1497 & 582.0483 & 767.9162 & 723.3138 & 723.4238 & 636.9478 \\
\hline
\end{tabular}

Table. 8 Variation Of Fault Indices With Distance At A Fault Inception Angle 40 And 100 Degrees For A-G Fault From Terminal-1

\begin{tabular}{|r|r|l|l|l|l|l|l|r|}
\hline $\begin{array}{l}\text { Distance; } \\
\text { Km }\end{array}$ & T1 & T2 & T3 & T4 & T1 & \multicolumn{1}{l|}{ T2 } & \multicolumn{1}{l|}{ T3 } & \multicolumn{1}{l|}{ T4 } \\
\hline 10 & 1059.681 & 544.0775 & 668.9153 & 498.1025 & 769.2924 & 387.0372 & 471.9511 & 376.8974 \\
\hline 20 & 1041.506 & 565.2126 & 682.5205 & 520.7857 & 756.7638 & 378.418 & 479.5775 & 370.2325 \\
\hline 30 & 1023.13 & 578.3497 & 708.9162 & 533.0858 & 741.4704 & 390.6652 & 496.9107 & 402.7711 \\
\hline 40 & 1006.601 & 586.3871 & 736.5021 & 537.3603 & 728.4823 & 441.9265 & 513.5268 & 451.262 \\
\hline 50 & 988.8552 & 604.0691 & 751.2017 & 556.9061 & 710.7219 & 471.799 & 528.5068 & 486.1536 \\
\hline 60 & 982.8408 & 624.7325 & 776.4945 & 578.8509 & 728.0993 & 493.3478 & 577.652 & 489.4027 \\
\hline 70 & 949.261 & 638.5712 & 791.3298 & 592.7429 & 665.3314 & 492.7726 & 558.8033 & 481.6643 \\
\hline 80 & 950.1704 & 648.1678 & 809.2706 & 598.7288 & 694.5508 & 466.2825 & 574.0523 & 456.511 \\
\hline 90 & 932.5459 & 655.0678 & 851.5977 & 609.681 & 686.6484 & 463.805 & 594.3207 & 461.1004 \\
\hline 100 & 913.1587 & 691.9791 & 869.1171 & 650.4348 & 661.97 & 497.7995 & 618.4955 & 511.1811 \\
\hline
\end{tabular}

Table. 9 Variation Of Fault Indices With Distance At A Fault Inception Angle 40 And 100 Degrees For A-B Fault From Terminal-1 
International Journal of Fuzzy Logic Systems (IJFLS) Vol.6, No.3, July 2016

\begin{tabular}{|r|l|r|l|l|l|l|l|l|}
\hline $\begin{array}{l}\text { Distance; } \\
\text { Km }\end{array}$ & T1 & T2 & T3 & T4 & \multicolumn{1}{l|}{ T1 } & \multicolumn{1}{l|}{ T2 } & T3 & T4 \\
\hline 10 & 1068.677 & 566.773 & 668.9154 & 535.3554 & 938.3116 & 462.8588 & 507.6697 & 436.5629 \\
\hline 20 & 1047.099 & 595.6038 & 682.5206 & 571.3748 & 911.257 & 481.7804 & 512.9274 & 453.7533 \\
\hline 30 & 1031.564 & 604.5939 & 708.9163 & 575.7132 & 883.7126 & 521.4633 & 524.6118 & 474.2159 \\
\hline 40 & 1017.387 & 603.689 & 736.5022 & 572.9129 & 854.1038 & 572.5719 & 574.6328 & 515.6368 \\
\hline 50 & 988.8551 & 608.6421 & 751.2017 & 561.2825 & 823.93 & 587.5612 & 603.5288 & 523.5786 \\
\hline 60 & 982.8407 & 621.1967 & 776.4946 & 579.9604 & 834.1278 & 595.4542 & 634.7603 & 517.0295 \\
\hline 70 & 949.261 & 656.3115 & 791.3299 & 626.9618 & 785.328 & 584.9426 & 605.6604 & 540.6712 \\
\hline 80 & 950.1703 & 667.2003 & 809.2707 & 618.662 & 783.2456 & 601.6417 & 635.3895 & 548.9573 \\
\hline 90 & 932.5458 & 681.4644 & 851.5906 & 655.273 & 782.3648 & 655.0769 & 682.0395 & 564.6995 \\
\hline 100 & 924.4261 & 679.3095 & 869.1155 & 663.7563 & 764.5291 & 670.2532 & 693.8669 & 596.0428 \\
\hline
\end{tabular}

Table. 10 Variation Of Fault Indices With Distance At A Fault Inception Angle 40 And 100 Degrees For A-B-G Fault From Terminal-1

\begin{tabular}{|r|l|l|l|l|l|l|l|l|}
\hline $\begin{array}{l}\text { Distance, } \\
\text { Km }\end{array}$ & T1 & T2 & T3 & T4 & T1 & T2 & T3 & T4 \\
\hline 10 & 995.9598 & 576.5083 & 649.9969 & 552.5509 & 1053.765 & 551.9506 & 654.2961 & 543.9325 \\
\hline 20 & 976.9548 & 581.5549 & 664.9678 & 548.2407 & 1037.499 & 561.1937 & 676.3916 & 537.5657 \\
\hline 30 & 962.2107 & 559.6292 & 680.3446 & 515.3753 & 1015.471 & 581.8696 & 699.5694 & 561.425 \\
\hline 40 & 947.4875 & 553.2859 & 691.473 & 512.2081 & 999.4461 & 602.0844 & 753.9723 & 578.1242 \\
\hline 50 & 933.0134 & 569.5216 & 710.4117 & 526.4373 & 979.3797 & 618.6817 & 768.1016 & 582.2014 \\
\hline 60 & 876.6351 & 582.619 & 780.3388 & 582.2767 & 962.9102 & 632.3221 & 773.6586 & 601.6078 \\
\hline 70 & 947.0886 & 646.0029 & 744.4863 & 638.6205 & 934.2452 & 646.6584 & 784.8537 & 625.7717 \\
\hline 80 & 895.6684 & 630.7086 & 787.3407 & 606.9076 & 923.3873 & 659.8792 & 781.3016 & 636.3791 \\
\hline 90 & 868.9065 & 665.7442 & 816.6199 & 615.9234 & 934.7853 & 651.8467 & 855.3063 & 652.9307 \\
\hline 100 & 902.4342 & 645.5892 & 849.1865 & 607.7717 & 933.2727 & 681.4823 & 850.6261 & 655.6692 \\
\hline
\end{tabular}

Table. 11 Variation Of Fault Indices With Distance At A Fault Inception Angle 40 And 100 Degrees For A-B-C Fault From Terminal-1

\begin{tabular}{|r|l|l|l|r|l|l|l|l|}
\hline \multicolumn{1}{|l|}{ Fia } & T1 & T2 & T3 & \multicolumn{1}{l|}{ T4 } & T1 & T2 & \multicolumn{1}{l|}{ T3 } & \multicolumn{1}{l|}{ 44 } \\
\hline 20 & 1219.143 & 700.0039 & 671.4878 & 594.539 & 1035.732 & 896.1634 & 882.9362 & 762.0628 \\
\hline 40 & 953.7788 & 552.7896 & 507.7162 & 501.401 & 831.3929 & 710.9502 & 675.0111 & 592.9964 \\
\hline 60 & 800.7785 & 498.0746 & 483.96 & 446.7802 & 692.6818 & 585.4767 & 585.1422 & 467.2439 \\
\hline 80 & 807.5176 & 447.7114 & 481.4047 & 365.0244 & 690.6542 & 611.4085 & 630.3941 & 458.1528 \\
\hline 100 & 973.8581 & 610.0538 & 632.7493 & 471.7781 & 853.2456 & 732.317 & 761.3227 & 524.4016 \\
\hline 120 & 1139.447 & 680.4346 & 654.9158 & 536.7472 & 959.0316 & 839.8206 & 830.0496 & 669.8206 \\
\hline 140 & 964.7391 & 581.0516 & 513.0762 & 508.741 & 849.1141 & 732.4191 & 697.6702 & 617.219 \\
\hline 160 & 797.9094 & 532.3816 & 475.7728 & 457.0477 & 700.0159 & 612.2244 & 607.309 & 508.2796 \\
\hline 180 & 785.6498 & 462.2793 & 446.3378 & 369.5129 & 683.0886 & 591.5452 & 621.6331 & 455.464 \\
\hline
\end{tabular}

Table. 12. Variation Of Fault Indices With Fault Inception Angle For A-G Fault At A Distance Of 30 And 90 Km From Terminal-1 


\begin{tabular}{|r|l|l|l|l|l|l|l|l|}
\hline \multicolumn{1}{|l|}{ Fia } & T1 & T2 & T3 & T4 & T1 & T2 & T3 & T4 \\
\hline 20 & 911.8137 & 518.5094 & 606.2 & 539.7431 & 816.0706 & 608.8137 & 718.3227 & 617.5568 \\
\hline 40 & 741.4704 & 390.6652 & 496.9107 & 402.7711 & 686.6484 & 463.805 & 594.3207 & 461.1004 \\
\hline 60 & 726.4182 & 393.8687 & 526.3431 & 382.3212 & 656.0156 & 449.4823 & 604.9852 & 428.7204 \\
\hline 80 & 856.1579 & 432.3557 & 617.6726 & 398.6446 & 790.5453 & 523.8051 & 730.0529 & 478.3351 \\
\hline 100 & 1023.13 & 578.3497 & 708.9162 & 533.0858 & 932.5459 & 655.0678 & 851.5977 & 609.681 \\
\hline 120 & 883.2645 & 536.4678 & 594.1763 & 533.5731 & 805.3385 & 615.8391 & 701.5673 & 619.166 \\
\hline 140 & 724.8093 & 424.5371 & 481.8222 & 429.233 & 664.3398 & 515.9693 & 589.8575 & 515.3548 \\
\hline 160 & 703.1744 & 419.8805 & 508.2027 & 407.555 & 645.5478 & 457.9211 & 586.2328 & 443.7543 \\
\hline 180 & 829.6808 & 445.337 & 594.6569 & 407.8118 & 756.382 & 533.5525 & 711.3583 & 498.3343 \\
\hline
\end{tabular}

Table. 13. Variation Of Fault Indices With Fault Inception Angle For A-B Fault At A Distance Of 30 And 90 Km From Terminal-1

\begin{tabular}{|r|l|l|l|l|l|l|l|r|}
\hline \multicolumn{1}{|l|}{ Fia } & T1 & T2 & T3 & T4 & T1 & T2 & T3 & \multicolumn{1}{l|}{ T4 } \\
\hline 20 & 1045.622 & 609.9567 & 633.9019 & 568.5621 & 911.7837 & 752.0754 & 806.0307 & 675.466 \\
\hline 40 & 883.7126 & 521.4633 & 524.6118 & 474.2159 & 782.3648 & 655.0769 & 682.0395 & 564.6995 \\
\hline 60 & 868.6603 & 524.6679 & 554.0449 & 453.7672 & 751.7264 & 640.8859 & 692.7108 & 532.3009 \\
\hline 80 & 990.0749 & 552.9266 & 638.5678 & 470.0897 & 871.5557 & 684.7021 & 789.7535 & 581.7934 \\
\hline 100 & 1031.564 & 617.7021 & 708.9163 & 575.7132 & 932.5458 & 703.67 & 851.5906 & 655.273 \\
\hline 120 & 1008.58 & 594.9839 & 643.4166 & 539.2599 & 892.2054 & 706.9534 & 801.2469 & 643.7637 \\
\hline 140 & 887.2006 & 523.5112 & 541.9808 & 475.0299 & 786.6489 & 641.3923 & 689.513 & 579.1409 \\
\hline 160 & 865.5657 & 518.8557 & 568.362 & 453.3531 & 767.8599 & 583.2894 & 685.8896 & 507.5822 \\
\hline 180 & 992.0721 & 544.3113 & 654.8155 & 453.6088 & 878.6909 & 658.5237 & 811.0333 & 562.2265 \\
\hline
\end{tabular}

Table. 14. Variation Of Fault Indices With Fault Inception Angle For A-B-G Fault At A Distance Of 30 And 90 Km From Terminal-1

\begin{tabular}{|r|l|r|l|r|l|l|l|l|}
\hline \multicolumn{1}{|l|}{ Fia } & T1 & T2 & T3 & T4 & T1 & T2 & T3 & T4 \\
\hline 20 & 1036.474 & 643.1597 & 754.6253 & 636.5044 & 975.4554 & 673.8964 & 886.7439 & 676.7351 \\
\hline 40 & 1015.471 & 581.8696 & 699.5694 & 561.425 & 934.7853 & 651.8467 & 855.3063 & 652.9307 \\
\hline 60 & 988.6142 & 584.2018 & 698.7609 & 565.0113 & 889.4003 & 664.8969 & 826.3877 & 656.4871 \\
\hline 80 & 979.4916 & 596.341 & 707.1953 & 562.3348 & 927.681 & 685.5562 & 825.4407 & 653.2122 \\
\hline 100 & 962.2107 & 559.6292 & 680.3446 & 515.3753 & 868.9065 & 665.7442 & 816.6199 & 615.9234 \\
\hline 120 & 981.2293 & 618.866 & 701.8045 & 576.9574 & 906.0513 & 670.0089 & 837.3046 & 629.1345 \\
\hline 140 & 980.8027 & 565.9892 & 682.6465 & 528.6996 & 891.8853 & 647.721 & 826.5214 & 625.2226 \\
\hline 160 & 1004.918 & 593.4622 & 705.3793 & 560.2411 & 900.2156 & 623.6577 & 822.6573 & 604.6658 \\
\hline 180 & 1013.016 & 582.095 & 726.5142 & 550.4384 & 899.0855 & 644.58 & 833.5269 & 634.2043 \\
\hline
\end{tabular}

Table. 15. Variation Of Fault Indices With Fault Inception Angle For A-B-C Fault At A Distance Of 30 And 90 Km From Terminal-1

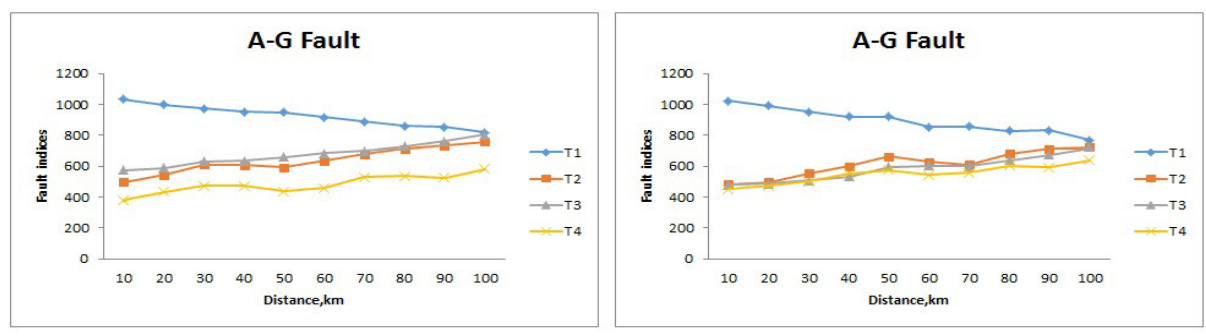

Fig. 11.Variation Of Fault Indices With Distance At A Fault Inception Angle Of 40 And 100 Degrees For A-G Fault From Terminal-1 

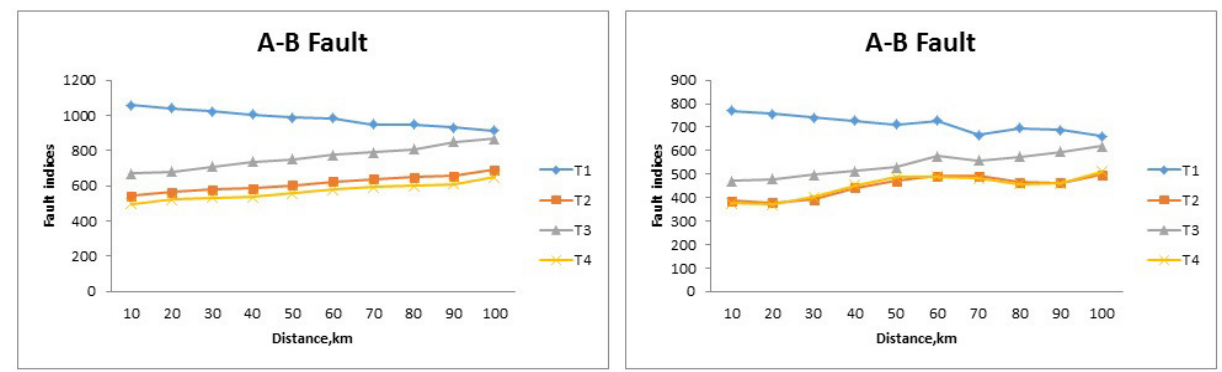

Fig. 12.Variation Of Fault Indices With Distance At A Fault Inception Angle Of 40 And 100 Degrees For A-B Fault From Terminal-1
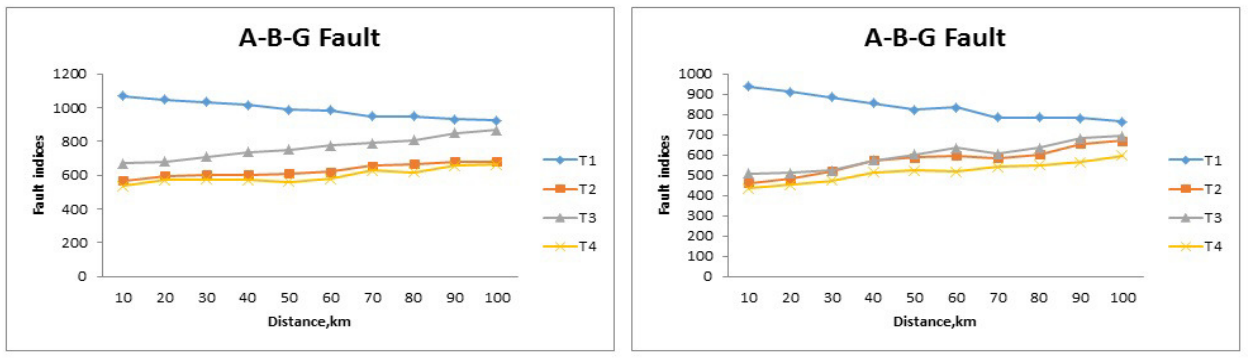

Fig. 13.Variation Of Fault Indices With Distance At A Fault Inception Angle Of 40 And 100 Degrees For A-B-G Fault From Terminal-1
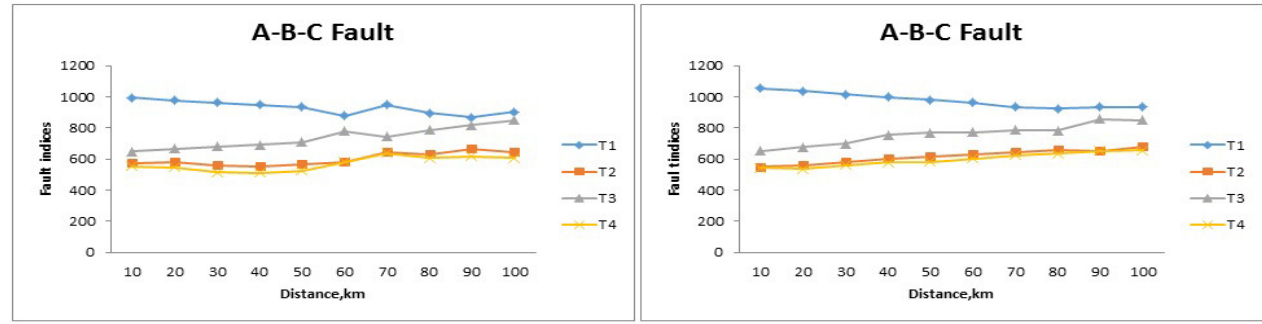

Fig. 14.Variation Of Fault Indices With Distance At A Fault Inception Angle Of 40 And 100 Degrees For A-B-C Fault From Terminal-1
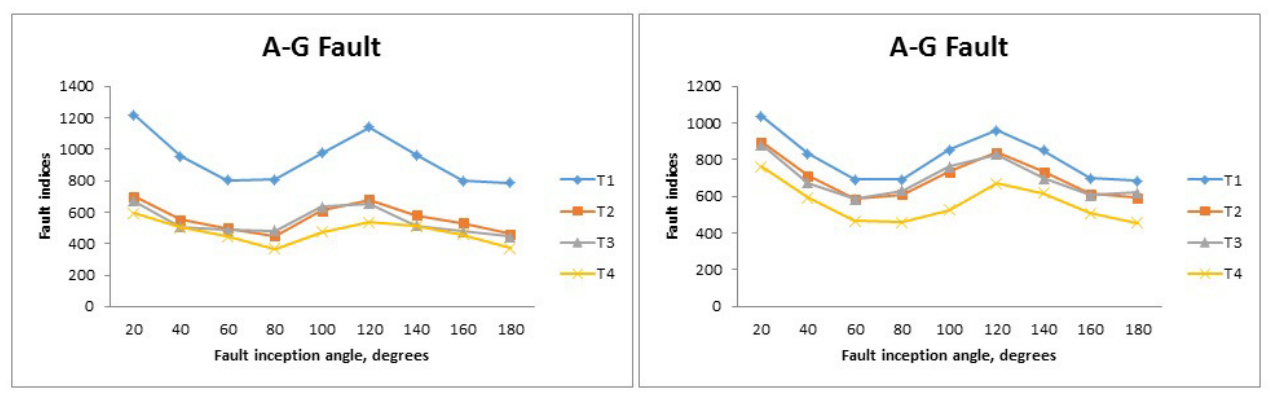

Fig. 15. Variation Of Fault Indices With Fault Inception Angle For A-G Fault At A Distance Of 30 And 90 Km From Terminal-1 

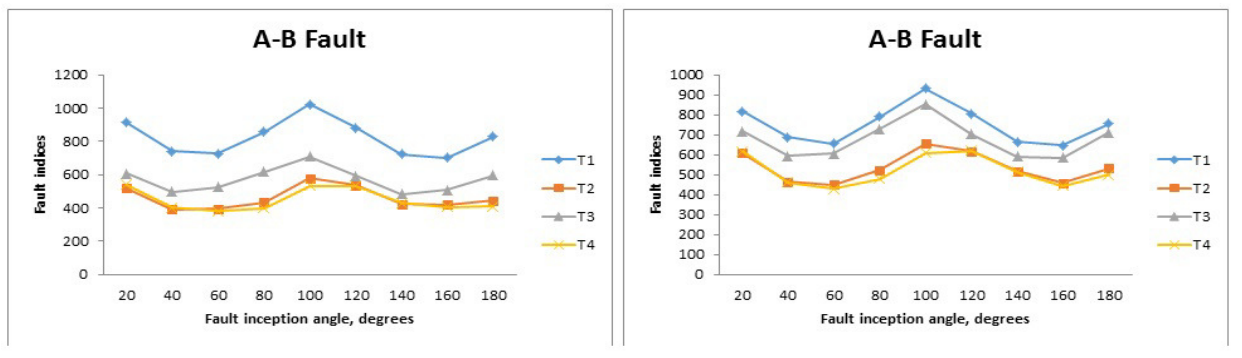

Fig. 16. Variation Of Fault Indices With Fault Inception Angle For A-B Fault At A Distance Of 30 And 90 Km From Terminal-1
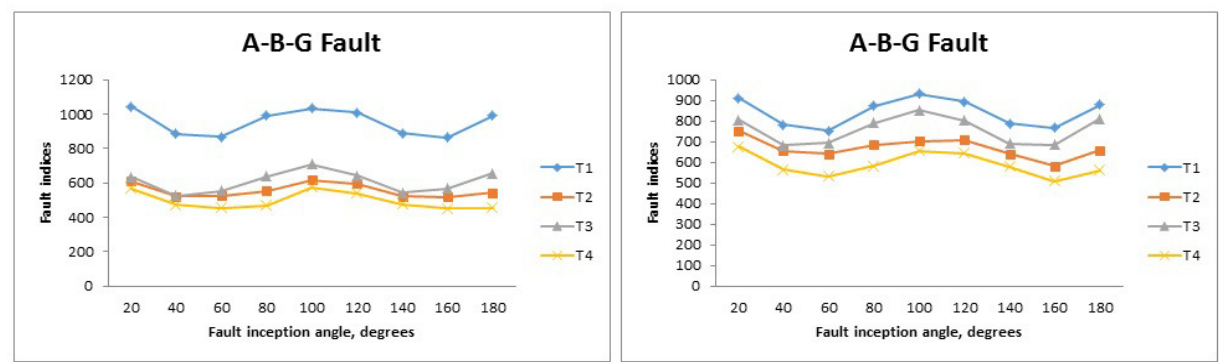

Fig. 17. Variation Of Fault Indices With Fault Inception Angle For A-B-G Fault At A Distance Of 30 And 90 Km From Terminal-1
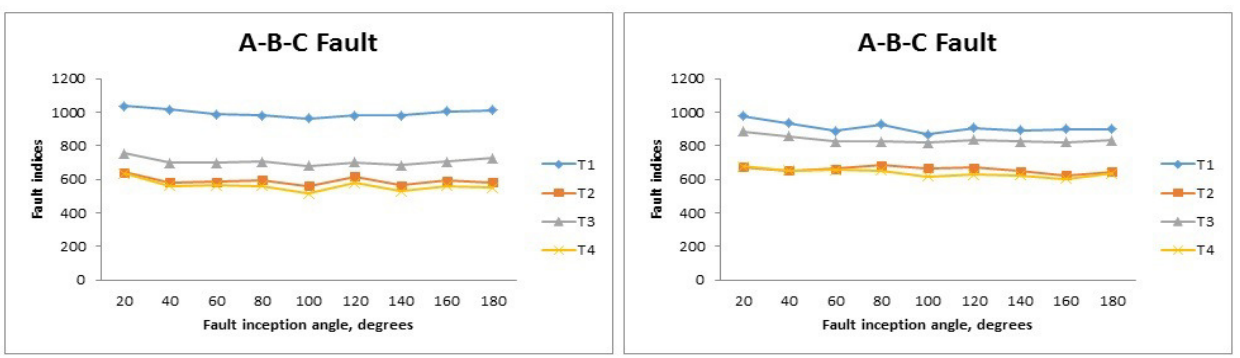

Fig. 18. Variation Of Fault Indices With Fault Inception Angle For A-B-C Fault At A Distance Of 30 And 90 Km From Terminal-1

Figures 4-7 Illustrate The Variation Of Fault Indices Of Three Phase Currents With Fault Inception Angle At A Distance Of $30 \mathrm{Km}$ And $90 \mathrm{Km}$ From Terminal-1 Towards The Path Of Terminal-1 To Terminal-2 For A-G, A-B, A-B-G, A-B-C Faults Respectively. Figures 8-11 Illustrate The Variation Of Fault Indices With Distance At A Fault Inception Angle $40^{\circ}$ And $100^{\circ}$ From Terminal-1 Towards The Path Of Terminal-1 To Terminal-2 For The Same Type Of Faults. In Both The Cases, It Is Observed That The Fault Indices Of Faulty Phase Is Large As Compared With That Of Healthy Phase. Thus The Number Of Faulty Phases Is Determined By Comparing The Fault Index With A Threshold Value (Th) Which Is Taken As 400. Once The Faulty Phase Is Identified, Then The Faulty Terminal Is Identified By Considering The Fault Indices Of That Particular Phase At All The Terminals And Comparing The Index Values At All The Terminals And The Index Value Of That Particular Phase At The Faulty Terminal Is Higher Than The Index Value Of The Same Phase At The Remaining Terminals. Figures 12-15 Illustrate The Variation Of Fault Index Of Current Of Phase 'A' At All The Terminals For A-G Fault, Fault Indices Of Phases A And B For A-B And A-B-G Faults, Fault Indices Of Phases Of A,B,C For A-B-C Fault At A Fault Inception Angle Of $40^{\circ}$ And $100^{\circ}$ With Variation In Distance From Terminal-1 
Towards The Path Of Terminal-1 To Terminal-2, It Is Observed That The Fault Indices Of That Particular Phase(S) At Faulty Terminal Is(Are) Higher As Compared With That Of Other Terminals. Figures 16-19 Illustrate The Variation Of Fault Index Of Current Of Phase 'A' At All The Terminals For A-G Fault, Fault Indices Of Phases A And B For A-B And A-B-G Faults, Fault Indices Of Phases Of A,B, C For A-B-C Fault At A Distance Of 30km And 90km From Terminal-1 Towards The Path Of Terminal-1 To Terminal-2, It Is Observed That The Fault Indices Of That Particular Phase(S) At Faulty Terminal Is(Are) Higher As Compared With That Of Other Terminals. Thus The Number Of Faulty Phases Are Determined By Comparing The Fault Index With A Threshold Value (Th) And The Faulty Terminal Is Identified By Comparing The Fault Index Of The Same Phase(S) At All The Terminals And The Faulty Terminal Fault Index Will Be Higher Than The Other Terminals For The Same Phase Which Identifies The Faulty Terminal.

\section{FAUlt Location Estimation}

After The Fault Detection, Classification And Faulty Terminal Identification, The Fault Location Has Been Estimated With Fuzzy Logic. For This Purpose, The Three Phase Current Signals At The Corresponding Terminal Have Been Decomposed With Bior 1.5 Mother Wavelet And The Fault Indices Obtained Were The Inputs To The Fuzzy Inference System. The Standard Fuzzy Membership Function Taken Was Triangular And The Output Function Taken Was Distance(D). Each Input Variable Is Quantized Into The Linguistic Variables Such As Very Low(Vl), Low(L), High(H), Very High(Vh) For The Universe Of Discourse Spanning From 0 To 1 And For The Output Variable, It Is The Length Of The Transmission System Divided Into Four Zones D1,D2,D3,D4.The Inputs Are Combined Together Based On The Expert Opinion And The Possible Rules Are Framed And The Output Is Defuzzified To Get The Crisp Value Of D. Simulations Were Carried Out Considering Variations In Fault Location And Fault Inception Angle For Different Types Of Faults.

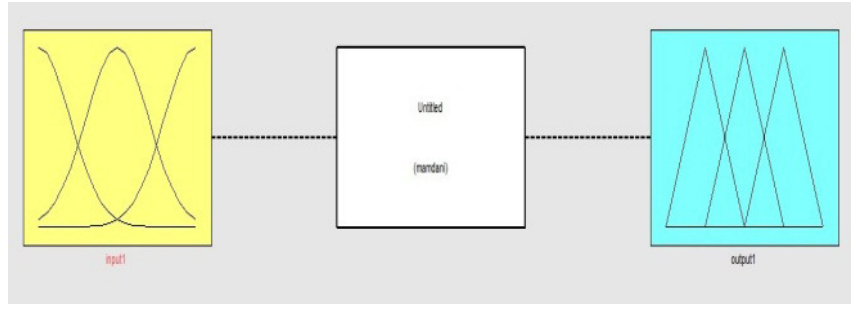

Figure 19: Fuzzy Inference System For Fault Location Estimation.

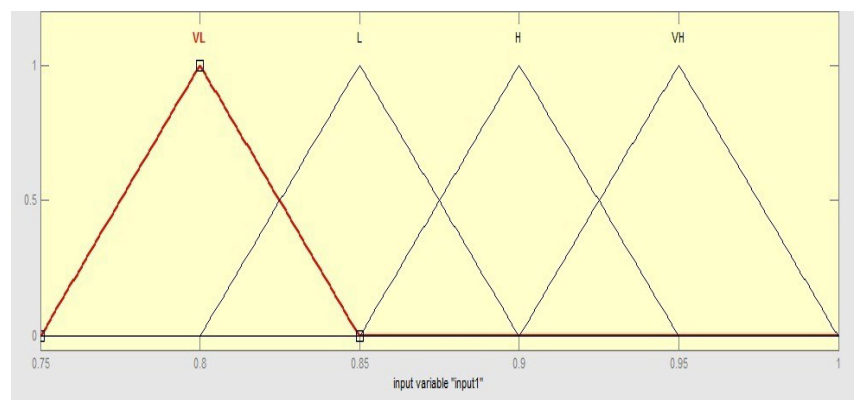

Figure 20: Input Variable (Fault Indices) For Fault Location From Terminal-1 


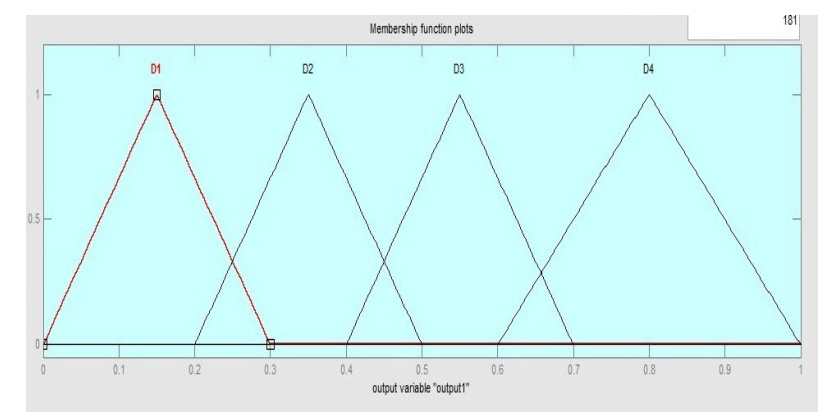

Figure 21: Output Variable For Location Of Distance

\begin{tabular}{|c|c|c|c|c|}
\hline \multirow[t]{2}{*}{ Fault Type } & \multirow{2}{*}{$\begin{array}{l}\text { Actual } \\
\text { Distance } \\
\text { From } \\
\text { Terminal-1 } \\
(\mathrm{Km})\end{array}$} & \multicolumn{3}{|c|}{ Transmission System } \\
\hline & & $\begin{array}{l}\text { Fuzzy } \\
\text { Distance, } \\
(\mathrm{Km})\end{array}$ & $\begin{array}{l}\text { Error } \\
\text { Distance }\end{array}$ & $\begin{array}{l}\% \\
\text { Error }\end{array}$ \\
\hline \multirow[t]{5}{*}{$A-G$} & 20 & 21.3 & 1.3 & 6.5 \\
\hline & 40 & 44.5 & 4.5 & 11.25 \\
\hline & 60 & 65.2 & 5.2 & 8.66 \\
\hline & 80 & 84.8 & 4.8 & 6 \\
\hline & 100 & 102.5 & 2.5 & 2.5 \\
\hline \multirow[t]{5}{*}{ A-B } & 20 & 21.2 & 1.2 & 6 \\
\hline & 40 & 44.3 & 4.3 & 10.75 \\
\hline & 60 & 64.4 & 4.4 & 7.3 \\
\hline & 80 & 85.14 & 5.14 & 6.4 \\
\hline & 100 & 104.6 & 4.6 & 4.6 \\
\hline \multirow[t]{5}{*}{ A-B-G } & 20 & 21.4 & 1.4 & 7 \\
\hline & 40 & 43.5 & 3.5 & 8.75 \\
\hline & 60 & 64.6 & 4.6 & 7.66 \\
\hline & 80 & 86.7 & 6.7 & 8.375 \\
\hline & 100 & 105.3 & 5.3 & 5.3 \\
\hline \multirow[t]{5}{*}{ A-B-C } & 20 & 21.6 & 1.6 & 8 \\
\hline & 40 & 42 & 2 & 5 \\
\hline & 60 & 65.4 & 5.4 & 9 \\
\hline & 80 & 84.3 & 4.3 & 5.375 \\
\hline & 100 & 106.5 & 6.5 & 6.5 \\
\hline
\end{tabular}

Table16: Fuzzy Based Fault Location Analysis From Terminal -1 Of The Transmission System

\section{Conclusions}

The Conventional Distance Relay Is Likely To Over Reach Or Under Reach Depending Upon The Mode, Type Of Facts Devices Incorporated In The Transmission System And Can Be Rectified By Wavelet Based Multi-Resolution Analysis Approach That Is Applied For Effective Fault Detection, Classification And Faulty Terminal Identification In Multi-Terminal Transmission Lines. The Above Algorithm Has Been Implemented For All Types Of Faults With Variations In Fault Inception Angle And Fault Distance And Location Of Statcom At All Terminals. The Results Indicate The Accuracy In Fault Detection, Classification And Faulty Terminal Identification, And Fault Location Estimation. This Scheme Is Proved To Be Unaffected By The Presence Of Statcom By Testing The Protection Scheme On Same Transmission System Without Statcom. The Proposed Protection Scheme Is Found To Be Fast, Reliable And Accurate For Various Types Of Faults On Transmission Lines With And Without Flexible Ac Transmission Control Device Such As Statcom At Different Locations And With Various Inception Angles. 


\section{REFERENCES}

[1] Amany M.El Zonkoly, Hussein Desouki, “Wavelet Entropy Based Algorithm For Fault Detection And Classification In Facts Compensated Transmission Line.” Energy And Power Engineering, Scientific Research,2011,Vol.3,Pp.34-42.

[2] Gorakshanath Abande, Mfar Satarkar,Mohan Thakre,V.S.Kale,Ganesh Patil "Impact Analysis Of Statcom On Distance Relay.” International Journal Of Innovative Research In Science, Engineering, Technology, Vol.3, Special Issue 3, March 2014.

[3] R.Illango, T.S.Rangeraja "Accurate Distance Protection Scheme For Transmission Line Fault Location Considering The Impact Of Shunt Connected Statcom”, Middle-East Journal Of Scientific Research, 21(11): 2123-2129,2014.

[4] Sriteja Alapati, B.Durga Prasad, "Effect Of Statcom On Distance Relay Performance In A Transmission Line", International Journal Of Computer And Electrical Engineering, Vol.4

[5] A.Y.Abdelaziz, Amr.M.Ibrahim “ A Hybrid Wavelet-Ann Based Protection Scheme For Facts Compensated Transmission Lines" International Journal Of Intelligent Sytems And Applications,2013,07,23-31

[6] Qazi Waqar Ali, Azzam Ul Asar, “ Smart Power Transmission System Using Facts Device”, International Journal Of Applied Power Engineering, Vol.2, No.2, August-2013, Pp.61-70.

[7] Sham M.V, Panduranga Vittal K “ Simulation Studies On The Distance Relay Performance In The Presence Of Statcom”, Journal Of Electrical Engineering.

[8] E.Kazeni Abharian, M.Karimi, P.Farhadi "Statcom Controller Design Based On Mlp For Power Flow Control”, International Journal Of Modeling And Optimization, Vol.1, No.4, October 2011, Pp.327333.

[9] R.Kameswara Rao, G.Ravikumar, Sheik Abdul Gafoor, S.S.Tulasiram "Fault Analysis Of Double Line Transmission System With Statcom Controller Using Neuro-Wavelet Based Techniques", International Journal Of Engineering Technology, Vol.2, No.6, June-2012

[10] Majid Jamil, Md.Abdul Kalam, A.Q.Ansari, M.Rizwan "Wavelet Ffnn Based Fault Location Estimation Of A Transmission Line" Electrical Engineering Research, Issue 3, July-2013.

[11] E.Latha Mercy, G.Jyosthna "Fault Detection And Classification In Transmission Lines Using Dwt And Anfis Techniques" Advanced Research In Electrical Engineering, Vol.2, No.2, OctoberDecember 2014, Pp.123-129.

[12] Bo, Z.Q., “A New Non-Communication Protection Technique For Transmission Lines”' Ieee Trans. Power Delivery,1998,13,(4), Pp. 1073-1078।

[13] Bhalija, B., And Maheswari, R.P,; "High Resistance Faults On Two Terminal Parallel Transmission Line; Analysis, Simulation Studies, And An Adaptive Distance Relaying Scheme”, Ieee Trans. Power Delivery, 2007, 22, (2), Pp. 801-812

[14] Brahma,S.M And Girgis,A..A. "Fault Location On A Transmission Line Using Synchronized Voltage Measurements', Ieee Transactions On Power Delivery, 2004,9(4),Pp. 1619-1622.

[15] Lyonette,D.R.M.,Bo,Z.Q, Weller G. And Jiang,G, “A New Directional Comparison Technique For The Protection Of Teed Transmission Circuits”. Power Engineering. Society, Winter Meeting, Ieee., January 2000, Vol.3, Pp. 1979-1984

[16] Bhalija,B.,And Maheswari, R.P; "New Differential Protection Scheme For Tapped Transmission Line', Iet Generation.Transmission,Distribution,2008,2,(2),Pp. 271-279

[17] Al-Fakhri,B, "The Theory And Application Of Differential Protection Of Multi-Terminal Lines Without Synchronization Using Vector Difference As Restraint Quantity-Simulation Study.' 8th Iee International Conference On Dpsp, April 2004, Vol.2, Pp. 404-409

[18] Yugant A.Parate, Ankita Y.Pai “ Power System Stability Improvement Using Facts Devices”, International Journal Of Engineering Research And General Science, Vol.3, Issue-5, Sep-Oct.2015

[19] Joe-Air Jiang, Ching-Shan Chen, Chih-Wenliu "A New Protection Scheme For Fault Detection, Direction Discrimination, Classification And Location In Transmission Lines". Ieee Transactions On Power Delivery, Vol.18, No.1, January-2003.

[20] Ching -Shan Chen, Chih-Wenliu "Fast And Accurate Fault Detection/Location Algorithm For Double Circuit/ Three Terminal Lines Using Pmus". Journal Of The Chinese Institute Of Engineering, Vol.26,No.3, 2003, Pp.289-299.

[21] Semwal, Vijay Bhaskar, Pavan Chakraborty, And Gora Chand Nandi. "Less Computationally Intensive Fuzzy Logic (Type-1)-Based Controller For Humanoid Push Recovery." Robotics And Autonomous Systems 63 (2015): 122-135. 


\section{AUTHORS}

J.Uday Bhaskar Received B.E From Gitam, Andhra University, Visakhapatnam. M.Tech From Jntu Kakinada.Presently Pursuing Ph.D From Jntu Kakinada. Research Areas Include Multiterminal Transmission Line Protection, Facts Devices.

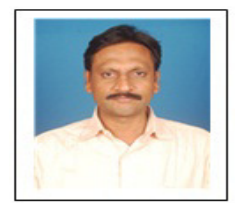

S.S Tulasi Ram Received B.Tech, M.Tech And Ph.D From Jntu College Of Engineering, Kakinada. Presently Working As Professor In The Department Of Electrical And Electronics Engineering In College Of Engineering, Jntuh, Hyderabad. His Areas Of Interest Include High Voltage Engineering, Power System Analysis And Control.

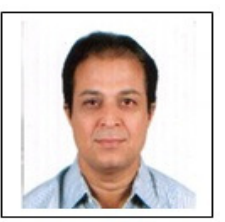

